\title{
UTILISATION DIGESTIVE ET RETENTION PAR LE PORC DU PHOSPHORE ET DU CALCIUM D'UN PHOSPHATE NATUREL DEFLUORE
}

PAR

\author{
G. CHARLET-LERY A. C. FRANCOIS A. M. LEROY ( $\left.{ }^{1}\right)$
}

Iaboratoire de Recherches de Zootechnie de l'Institut National Agronomique

\section{PLAN DU MEMOIRE}

Introduction.

Protocole expérimental.

Résultats.

'Teneur des fecès et de l'urine en phosphore et en calcium.

Digestibilité, bilan physiologique et retention du $\mathrm{P}$ et du $\mathrm{Ca}$

Interprétation et discussion.

Fixation quotidienne de phosphore et de calcium.

Résumé et conclusions.

\section{INTRODUCTION}

L'utilisation physiologique du phosphore et du calcium en fonction de la substance organique ou minérale qui apporte ces deux éléments a fait l'objet de nombreux travaux. En ce qui concerne le calcium, citons, en particulier JACQUOT et Collaborateurs (I) qui classent les différents sels de calcium selon leur bilan d'utilisation $\frac{\mathrm{Ca} \text { Ingéré }- \text { (Ca fécal }+\mathrm{Ca} \text { urinaire) }}{\text { Ca Ingéré }}$ dans l'ordre décroissant suivant : acétylgluconate, phosphate tricalcique, lactate, carbonate, phosphate d'éthyle et de calcium, gluconate, glycérophosphate, sulfate; toutefois le rapport $\frac{\mathrm{Ca}}{\mathrm{P}}$ de ces différents sels varie de 0,644 à $I, 8$ et ces variations ont pu influencer les bilans.

Par ailleurs, l'utilisation du calcium n'est pas perturbée par la présence de magnésium. RANDOrn et CAUSERET (2) ne trouvent, en effet,

(') Avec l'assistance technique de Mme Bozzi et M. G. Gauch, Melle J. Pinet. 
aucune différence caractéristique entre l'utilisation du calcium du calcaire dolomitique ou du carbonate de calcium pur par le rat.

De nombreux sels des acides phosphoriques sont assimilables : phosphate tricalcique, monosodique, potassique, pyrophosphate de sodium, etc. Les sels de calcium de l'acide orthophosphorique présentent un très grand intérêt alimentaire. En particulier, les poudres d'os et les phosphates de calcium d'origine minérale sont utilisés pour l'alimentation des animaux. Toutefois, l'utilisation des phosphates naturels est souvent limitée par leur teneur élevée en fluor. L'influence de cet élément sur l'utilisation du calcium par l'animal ressort nettement des travaux de MC CluRE et MitChELI (3) : 1orsque le taux de fluor contenu dans du phosphate tricalcique croît (de 0,5 à 3,8\%), les balances de calcium des porcs en expériences diminuent et les gains journaliers de poids vif décroissent.

Les phosphates d'Afrique du Nord sont souvent riches en fluor. Pour cette raison, leur utilisation pour l'alimentation des animaux est limitée. Par exemple, Pero (4) expérimentant sur le porc, conclut que le phosphate de Gafsa contenant $2,4 \%$ de fluor et $7,3 \%$ de silice, ne peut être utilisé directement.

Certains procédés industriels permettent d'éliminer le fluor des phosphates naturels. L'un de ceux-ci nouvellement mis au moint, permet d'obtenir un produit presque entièrement débarrassé de ce métalloïde, et, par voie de conséquence, éventuellement utilisable pour 1'alimentation animale.

Dans le présent mémoire, nous étudions l'utilisation du phosphore et du calcium de ce phosphate par le porc en croissance. La mesure des quantités d'alimentsingérés et des quantités de fèces et d'urine rejetés d'une part, le dosage de $\mathrm{P}$ et $\mathrm{Ca}$ dans chacun de ces milieux, d'autre part, permettent d'établir les coefficients d'utilisation digestive et de rétention de ces deux éléments, ainsi que les bilans d'utilisation physiologique.

\section{PROTOCOLE EXPÉRIMENTAL}

\section{A. - Animaux :}

Trois jeunes porcs Large White, pesant respectivement 28,30 et $34 \mathrm{~kg}$ au début de l'essai sont soumis à l'expérience. Ces animaux - en bon état de santé - proviennent du Centre National de Recherches Zootechniques de Jouy-en-Josas.

\section{B. - Alimentation :}

a) Aliment DE base:

La composition de l'aliment consommé par les animaux pendant les diverses périodes expérimentales est la suivante: Orge, $30 \%$, Manioc 
$35 \%$, Avoine ro $\%$, Tourteau de soya $5 \%$, Farine de viande $3 \%$, Babeurre $\sec 4 \%$, Levure de distillerie $8 \%$, Tourteau de lin $2 \%$, Farine de luzerne $3 \%$.

L'analyse de cet aliment donne les résultats suivants: Matière sèche : $87,78 \%$, Matières minérales : 3,98\%, Matières azotées $(\mathrm{N} \times 6.25)$ : I5,93 \%, Matières grasses : $3,43 \%$, Matières cellulosiques : $6,25 \%$, Extractifs non azotés : 58, I9 \%, Matières protéiques digestibles : I3,0 \%.

Un pareil mélange correspond aux besoins des porcs en croissance, âgés d'environ 4 mois. Il est distribué sous forme liquide. A cet aliment est ajouté I \% de carbonate de calcium pur, afin de ramener le rapport $\frac{\mathrm{Ca}}{\mathrm{P}}$ à une valeur voisine de 2 .

Les animaux reçoivent chaque jour, sous forme d'huile concentrée, 2500 U.I. de vitamine $A$ et $45^{\circ}$ U.I. de vitamine $D_{3}$.

\section{b) Phosphate DÉFLUORÉ :}

La composition du phosphate est la suivante : P I8,43\%, Ca 22, I0 \%, $0,073 \%, \mathrm{Na} \mathrm{I} 4,55 \%$, Insoluble chlorhydrique $6,6, \%$, Fluor 0,035\%, présence de magnésium, d'aluminium, de fer.

Mis en suspension à raison de $3 \%$ dans de l'eau distillée, il donne une solution surnageante de $\mathrm{pH}$ égal à 8,7 . Peut-être faut-il voir, dans cette alcalinité, la raison de l'inappétence qui a été observée, comme nous le verrons plus loin, au cours de l'une des périodes exprimentales (Pêriode D).

Le rapport $\frac{\mathrm{Ca}}{\mathrm{P}}$, égal à $\mathrm{I}, 2$, diffère du rapport phospho-calcique du phosphate tricalcique. En effet, le produit provient de l'application à un phosphate naturel d'un traitement chimique qui a pour effet de remplacer une partie du calcium par du sodium et d'éliminer à peu près totalement le fluor.

\section{C. - Méthodes de dosage du phosphore et du calcium :}

a) MINÉRALisation :

Nous utilisons la méthode de minéralisation nitroperchlorique, qui permet de doser, sur les mêmes liqueurs, le phosphore et le calcium.

Par exemple, pour les aliments et les fèces, I à $2 \mathrm{~g}$ de produit sont attaqués par $25 \mathrm{cc}$ de mélange nitro-perchlorique (2 $500 \mathrm{cc}$ d'acide nitrique et I $\mathrm{kg}$ d'acide perchlorique). Une dilution convenable est effectuée afin d'obtenir environ 0,2 à $0,5 \mathrm{mg}$ de phosphore par prise.

Pour l'urine, une prise de 25 à 50 cc d'urine est minéralisée dans les mêmes conditions.

Après ajustage à $200 \mathrm{cc}$, le dosage est effectué sur 5 ou ro cc. 


\section{b) CALCIUM :}

Le calcium est dosé selon la méthode de CAHN, Houget et JACQUOT (5). Il est précipité à $\mathrm{pH} 4,6$ par une solution d'acide oxalique à $9 \%$. Le titrage s'effectue à l'aide d'une solution de permanganate de $\mathrm{K} 0,02 \mathrm{~N}$

\section{c) PHOSPHORE :}

Le phosphore total est dosé à l'aide de la méthode de Thrvolue (6), dont la technique est la suivante : on précipite d'abord le phosphomolybdate d'ammonium. Le précipité filtré, lavé, est dissous dans une solution de soude étendue. On réduit alors le molybdate par le zinc en milieu acide, et l'on effectue un titrage molybdo-manganimétrique du sesquioxyde de molybdène.

A la place du microfiltre proposé par THrvolLE, nous utilisons des filtres de verre fritté de porosité $n^{\circ} 4$.

Des dosages effectués sur des solutions témoins nous ont montré que ces techniques sont satisfaisantes.

\section{D. - Mesures :}

Le protocole expérimental suivi a été décrit dans une publication précédente de deux d'entre nous (7). Les animatux sont pesés tous les matins à jeun. Nous prélevons des échantillons d'aliment après humidification ; ainsi, le calcium contenu dans l'eau est dosé avec celui provenant de l'aliment.

Nous mesurons journellement les ingestions, ainsi que les excrétions fécales et urinaires des trois animaux, et nous établissons :

a) le coefficient d'utilisation digestive ou coefficient de digestibilité :

$$
\text { C.U.D. }=\frac{\text { élément ingéré }- \text { élément fécal }}{\text { élément ingéré }} \times \text { Ioo }
$$

La valeur du C.U.D. est déterminée pour la matière sèche, le phosphore, le calcium, et dans certains cas, pour les matières minérales totales.

b) le bilan d'utilisation physiologique du phosphore et du calcium, égal au rapport :

$$
\frac{\text { élément ingéré }- \text { (élément fécal }+ \text { élément urinaire) }}{\text { élément ingéré }} \times \text { Ioo }
$$

c) le coefficient de rétention du phosphore et du calcium, égal au rapport :

$$
\frac{\text { élément ingéré }- \text { (élément fécal }+ \text { élément urinaire) }}{\text { élément ingéré }- \text { élement fécal }} \times \text { roo }
$$




\section{E. - Périodes expérimentales. Prélèvements.}

Pendant une période préliminaire, les animaux consomment l'aliment dont la composition est indiquée précédemment, additionné de I \% de carbonate de calcium pur. Pendant les périodes suivantes, nous ajoutons à l'aliment un composé minéral dans lequel le calcium et le phosphore sont apportés exclusivement par le phosphate défluoré étudié. La composition de ce mélange est la suivante :

Phosphate défluoré 75, Chlorure de sodium 55 , Carbonate de magnésium 6,7 , Sulfate ferreux 3 , Sulfate de cuivre 0,3 .

Au cours des différentes périodes, le composé minéral est incorporé dans 1'aliment d'abord au taux de $3 \%$ (soit $2,25 \%$ de phosphate) puis de $6 \%$ (soit $4,5 \%$ de phosphate).

Au cours des différentes périodes, nous étudions d'une part les ingestions et les excrétions fécales et urinaires quotidiennes de phosphore et de calcium (périodes $\mathrm{A}, \mathrm{B}, \mathrm{D}, \mathrm{F}$ ). Ces prélèvements quotidiens nous permettent de suivre les variations d'utilisation digestive et de rétention de $\mathrm{P}$ et de $\mathrm{Ca}$ consécutives aux modifications que nous apportons dans la teneur du régime en ces éléments. D'autre part, au cours des périodes $\mathrm{C}$ et $\mathrm{E}$, des échantillons moyens sont constitués pour chaque animal en réunissant les parties aliquotes des prélèvements quotidiens correspondants. Ces périodes se succèdent dans l'ordre suivant:

\begin{tabular}{|c|c|c|c|}
\hline & Durée & Régime & Analyse des échantillons \\
\hline A & 7 jours. & Témoin & Chaque jour, les 5 premiers jours. \\
\hline B & 5 jours. & Témoin $+3 \%$ minéral & Chaque jour. \\
\hline C & 8 jours. & Témoin $+3 \%$ minéral & Echantillon moyen. \\
\hline$D$ & 5 jours. & Témoin $+6 \%$ minéral & Chaque jour. \\
\hline $\mathrm{E}$ & 6 jours. & Témoin $+3 \%$ minéral & $\begin{array}{l}\text { Échantillon moyen les jours } 3-4-5 \text {. } \\
\text { Echantillon individuel jour } 6 \text {. }\end{array}$ \\
\hline $\mathrm{F}$ & 3 jours. & Témoin (sans $\mathrm{CO}_{3} \mathrm{Ca}$ ) & Chaque jour. \\
\hline
\end{tabular}

La période $D$ n'a pu être pourstrivie comme nous en avions l'intention, en raison du manque d'appétit manifesté par les animaux à partir du troisième jour de cette période. I es consommations quotidiennes n'atteignaient, en effet, que $70 \%$ de celles de la période précédente. L'appétit est revenu lorsque la teneur en mélange minéral de l'aliment distribué a été ramenée à $3 \%$ (Régime $\mathrm{E}$, analogue aux régimes $\mathrm{B}$ et $\mathrm{C}$ ).

\section{RESULTATS}

Les teneurs en phosphore et en calcium des ingestas correspondant aux différentes périodes, sont indiquées dans le tableau I. La difficulté d'obtenir un mélange parfaitement homogène, ainsi que les variations 
éventuelles de la teneur en calcium de l'eau ajoutée (31 par kg d'aliment) expliquent les variations enregistrées pour des régimes identiques.

TABLEAU I

en $\mathrm{g} / \mathrm{kg}$ d'aliment.

\begin{tabular}{|c|c|c|c|}
\hline Période & $\mathrm{P}$ & $\mathrm{Ca}$ & $\overline{\mathrm{P}}$ \\
\hline$\ldots \ldots \ldots, \ldots, \ldots, \ldots$ & $\overline{5,12}$ & II & 2,18 \\
\hline$\ldots \ldots \ldots \ldots \ldots \ldots+\ldots$, & 8,14 & 16,44 & 2,02 \\
\hline $\mathrm{C} \ldots \ldots$ & 8,35 & 14,25 & $\mathrm{r}, 7 \mathrm{I}$ \\
\hline $\mathrm{D} \ldots \ldots \ldots \ldots \ldots \ldots \ldots \ldots \ldots \ldots \ldots \ldots$ & IO,I I & 17,96 & 1,78 \\
\hline $\mathrm{E} \ldots \ldots \ldots \ldots \ldots \ldots \ldots$ & 7,89 & 15,03 & 1,90 \\
\hline $\mathrm{F}, \ldots \ldots \ldots, \ldots, \ldots, \ldots$ & 4,44 & 8,55 & 1,92 \\
\hline
\end{tabular}

Les tableaux IV, $\mathrm{V}$ et VI indiquent les bilans de $\mathrm{P}$ et $\mathrm{Ca}$ correspondant à chacun des animaux.

La croissance des animaux n'a pas présenté d'anomalies pendant toute la durée de l'expérience (voir tableau VIII) sauf lorsque le phosphate a provoqué de l'inappétence.

\section{IV. - INTERPRETATION ET DISCUSSION}

\section{a) Teneur des fèces et de l'urine en phosphore et en calcium.}

La teneur des matières fécales en phosphore et en calcium au cours de chacune des périodes expérimentales présente une bonne régularité (tableau II). Les variations journalières correspondant à chaque animal sont, en effet, relativement faibles. Au cours des périodes comportant l'addition de phosphate défluoré à la ration, on constate un rapide accroissement des taux de $\mathrm{P}$ et $\mathrm{Ca}$ des fèces (fig. I). Inversement, la décroissance des teneurs suit rapidement le retour du régime exempt de phosphate défluoré.

En revanche, le coefficient d'utilisation digestive subit de très fortes variations journalières (tableau IV, V et VI). Le tableau IV - particulièrement typique - permet de constater que ces variations sont parallèles à celles que subit la digestibilité de la matière sèche. Or, les différences relatives au $\mathrm{P}$ et au Ca sont amplifiées en raison des faibles taux de ces éléments dans les ingestas et les excrétas.

Toutefois, ces différences journalières se compensent et les digestibilités moyennes, correspondant à chaque période, sont très voisin es. I1 n'est pas possible de tenir compte de la période $D$, qui a provoqué de l'inappétence. Il n'est toutefois pas exclu que cette inappétence soit due à un autre constituant minéral que le phosphate défluoré. Cependant, pour aucun d'entre eux, nous n'avons dépassé les doses couramment recommandées par les normes usuelles. 


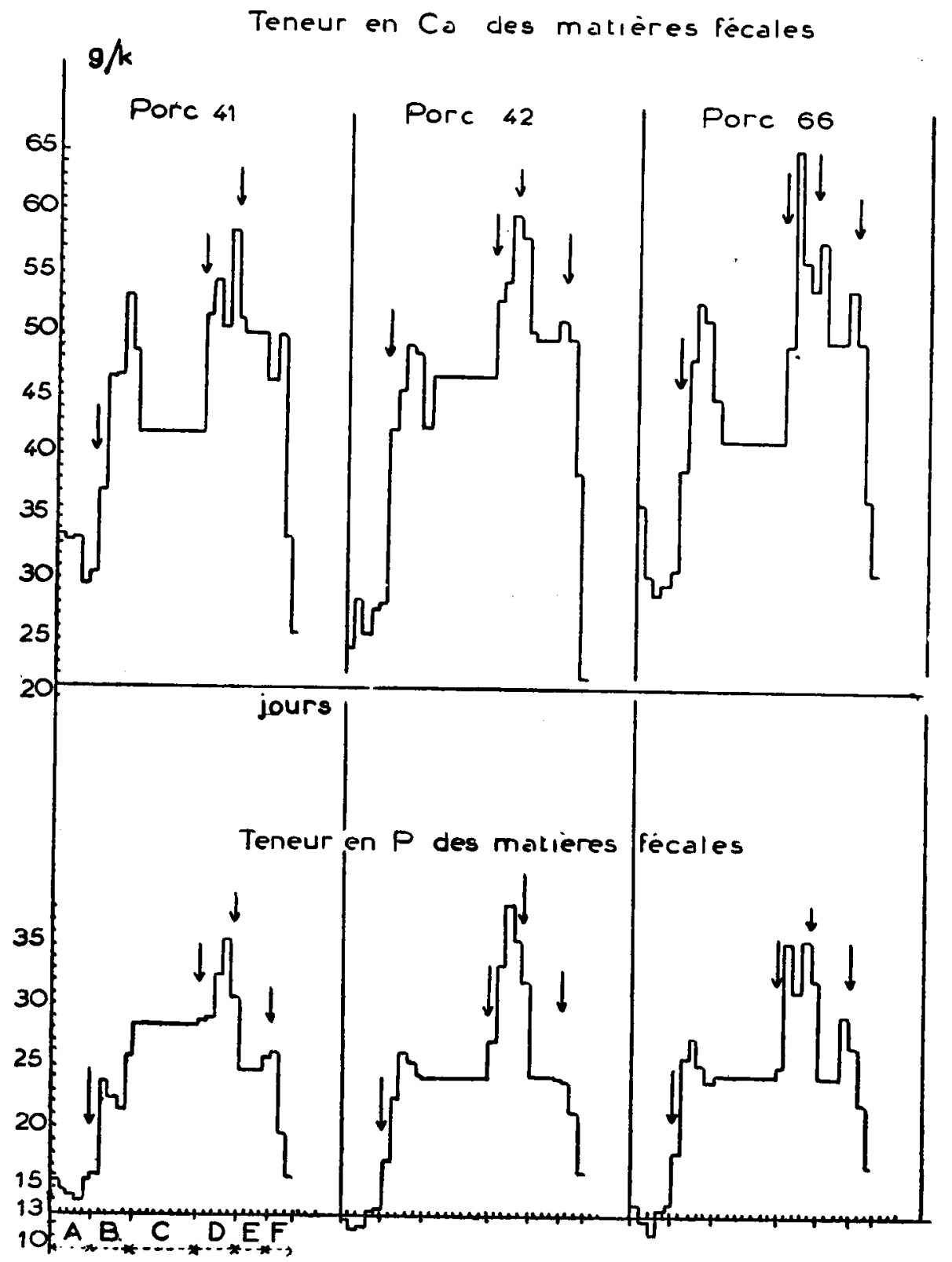

Fig I. 
C. CHARLET-LERY, A.-C. FRANÇOIS, A.-M. LEROY (IV, I953)

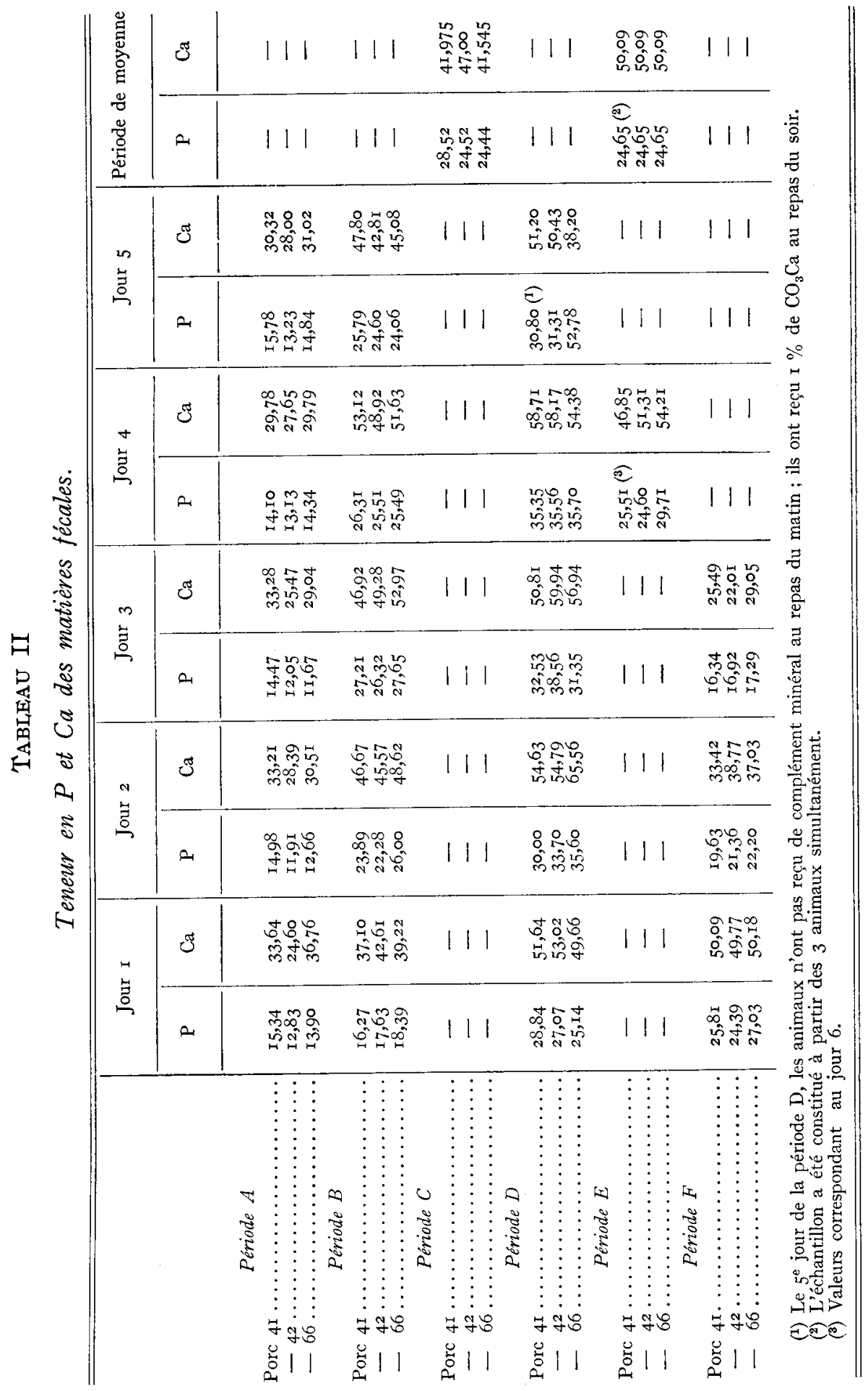


(IV, I953) UTILISATION DIGESTIVE DU PHOSPHORE ET DU CALCIUM 293

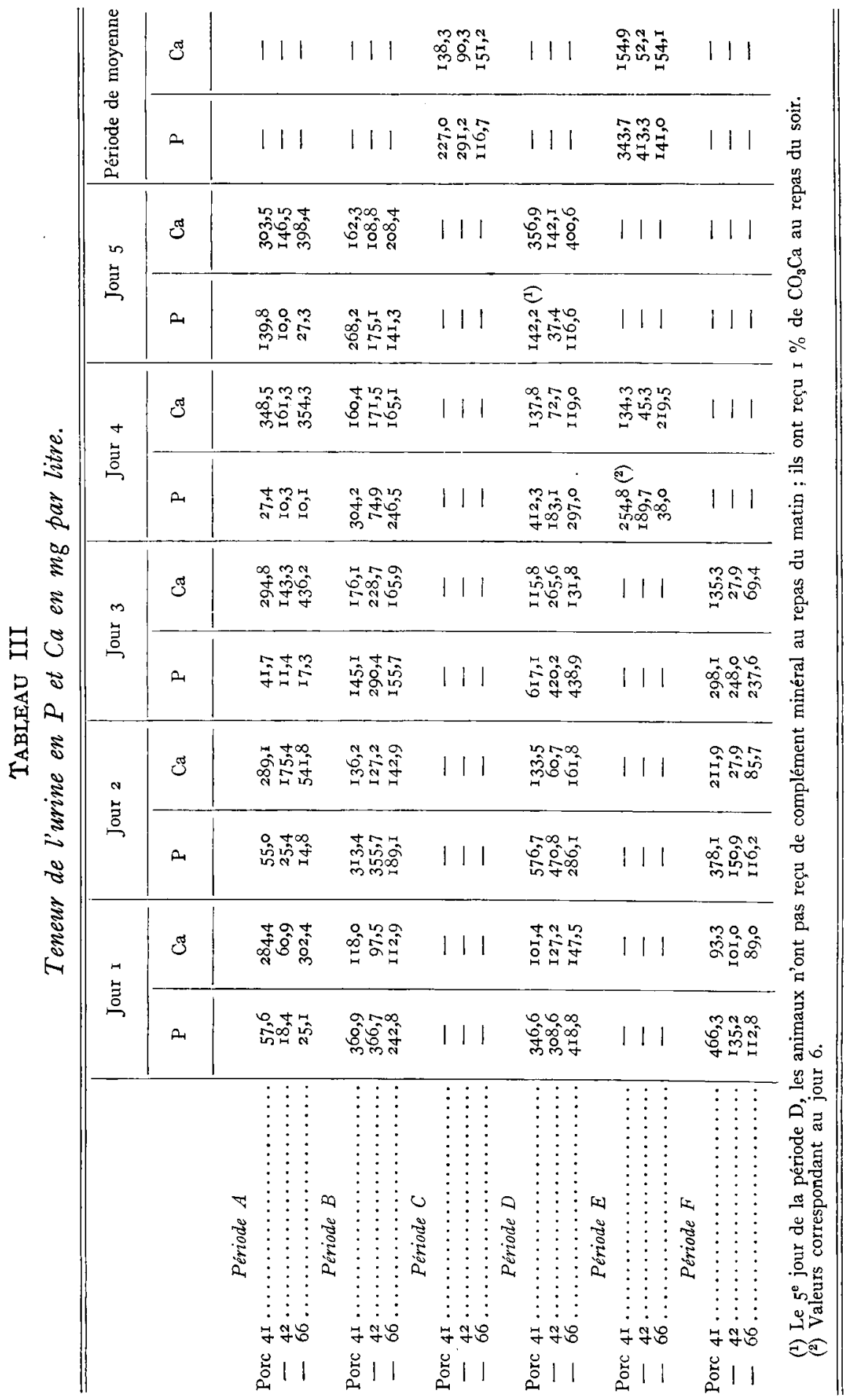




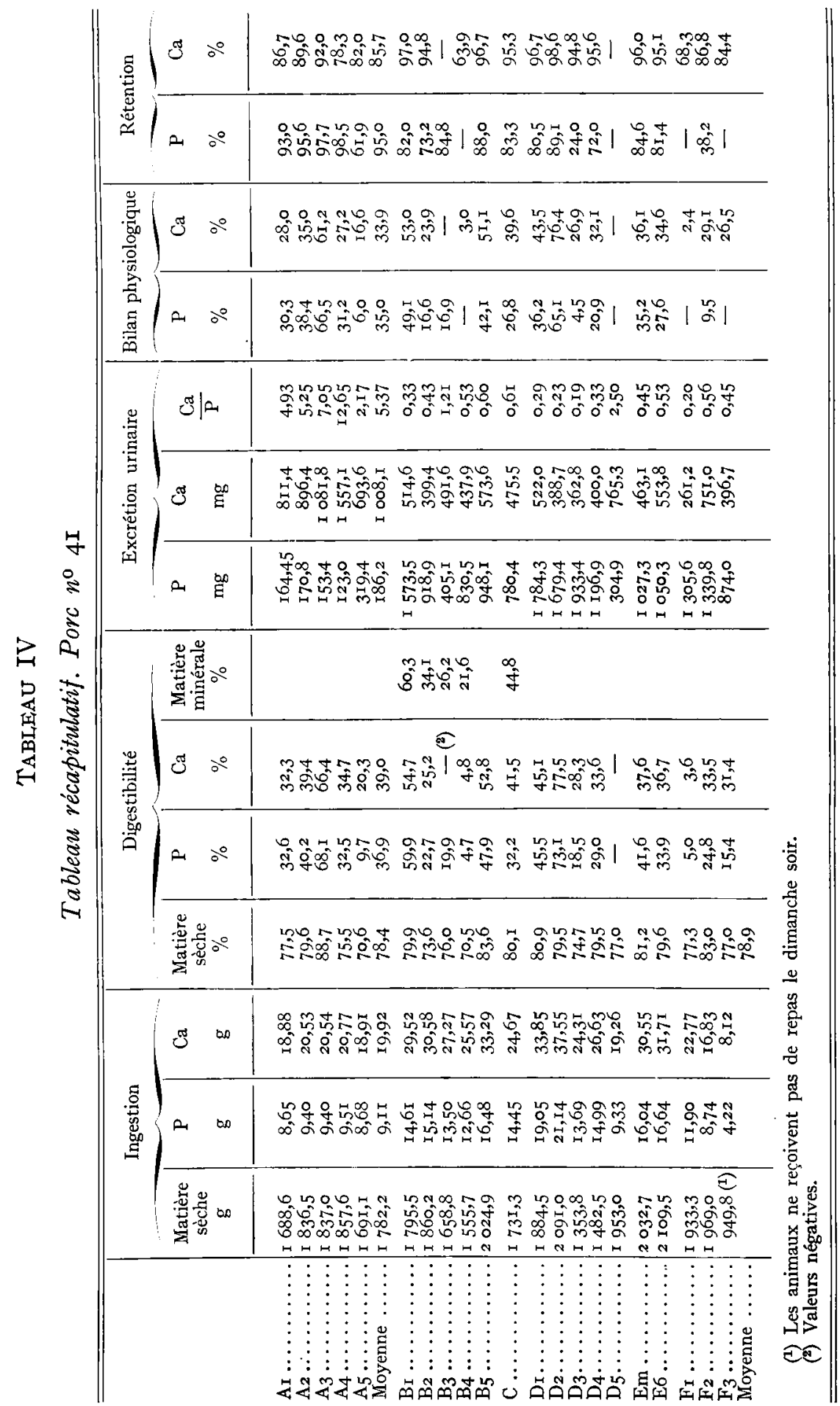


(IV, I953) UTILISATION DIGESTIVE DU PHOSPHORE ET DU CALCIUM 295

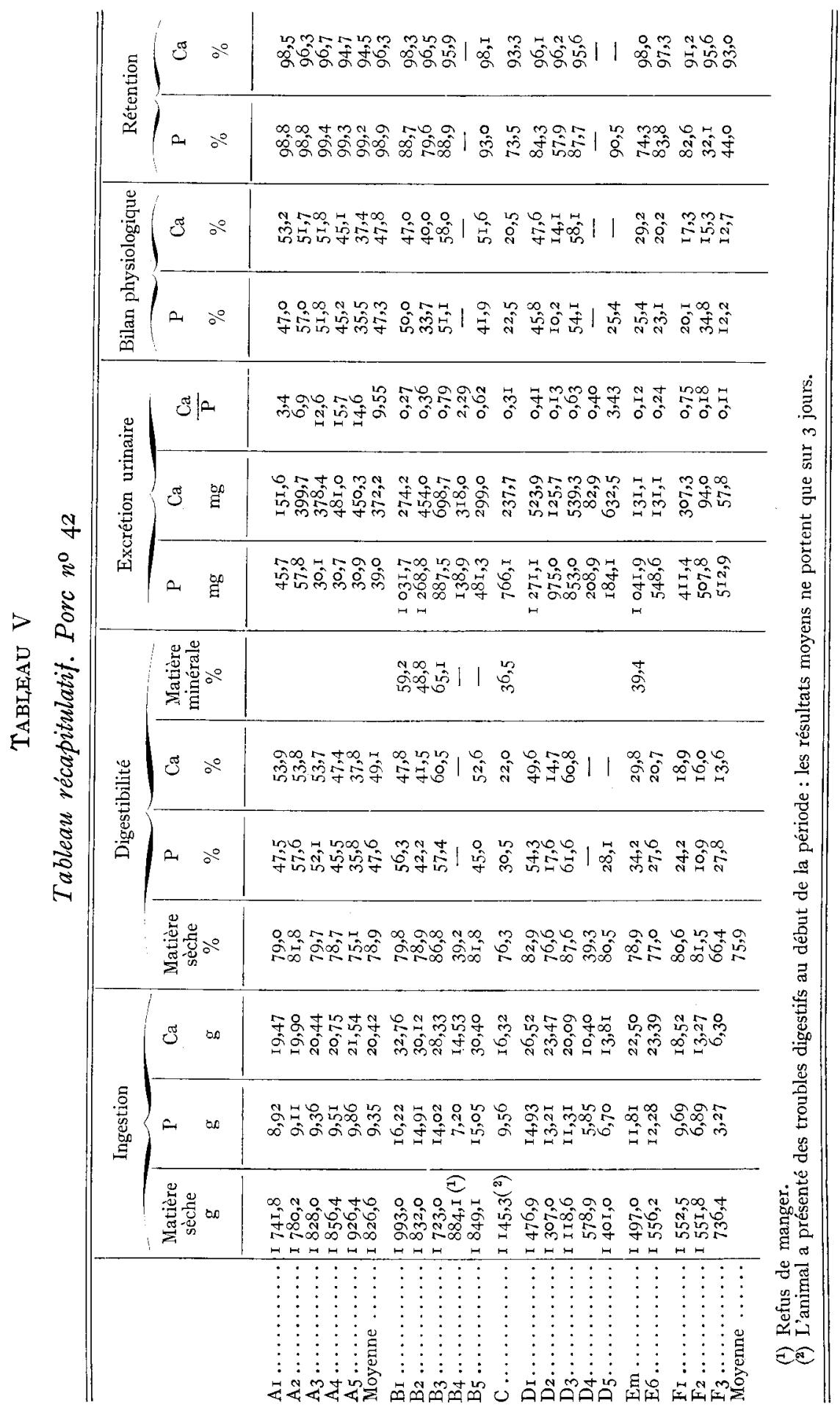




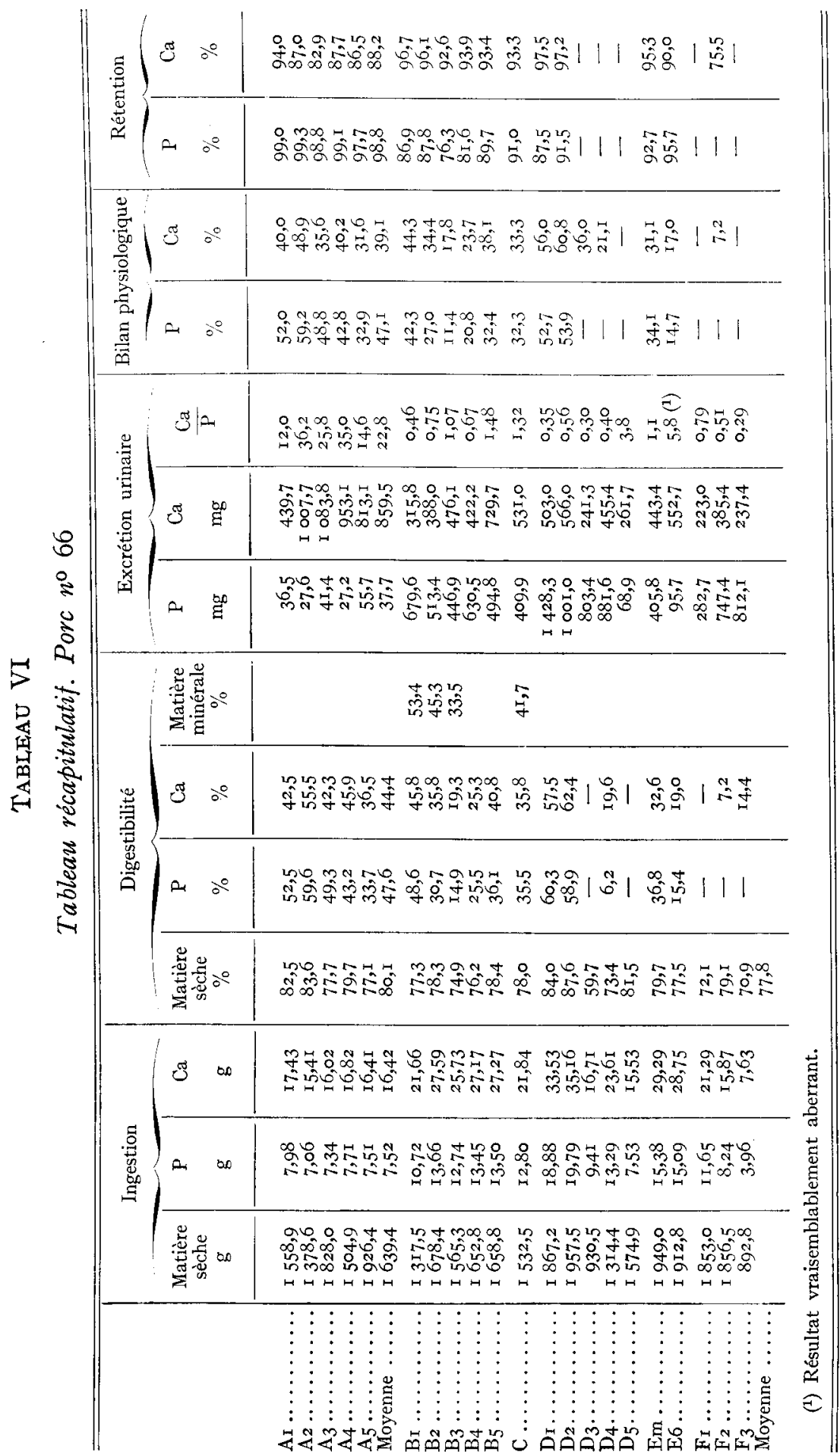




\section{TABLEAU VII}

\section{Coefficients moyens d'utilisation digestive}

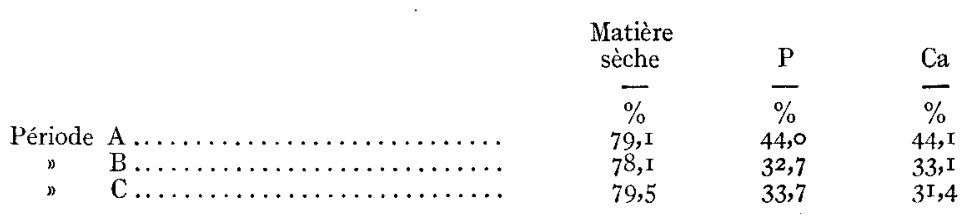

Nous constatons, ainsi que Pero (4), que l'utilisation du phosphore et du Calcium d'un régime entichi de phosphate est moins élevée que celle qui correspond an régime de base.

Toutefois, Bachmann et collaborateurs (8) ont montré que 1'utilisation du $\mathrm{P}$ et du Ca du régime par le rat, varie en raison inverse du taux de ces éléments dans la ration. Il n'est donc pas possible de conclure à une utilisation insuffisante du phosphore et de calcium du phosphate défluoré.

Les taux de phosphore et de calcium de l'urine présentent des variations beaucoup plus considérables que ceux des fèces (tableau III). Le rapport $\frac{\mathrm{Ca}}{\mathrm{P}}$ correspondant à l'élimination urinaire quotidienne varie en particulier très rapidement lorsque l'on modifie les teneurs du régime en phosphore et en calcium. Lorsque l'apport minéral est faible (période A) le rapport $\frac{\mathrm{Ca}}{\mathrm{P}}$ urinaire est élevé : sa valeur est comprise entre 5 et 36 . L'animal $n^{\circ} 66$ présente un rapport particulièrement élevé (tableaux IV, $\mathrm{V}$ et $\mathrm{VI}$ ). Lorsque le régime est enrichi simultanément en $\mathrm{Ca}$ et $\mathrm{P}$, les valeurs du rapport entre ces deux éléments s'abaissent très rapidement : dès le lendemain de la modification du régime, la variation est très nette. On observe alors un rapport $\frac{\mathrm{Ca}}{\mathrm{P}}$ inférieur à $I$. Ainsi, il apparaît que le phosphore ingéré est très rapidement excrété. En effet, la comparaison des bilans physiologiques moyens et des coefficients de rétention moyens met ce fait en évidence.

\begin{tabular}{|c|c|c|c|c|}
\hline & \multicolumn{2}{|c|}{ Bilan physiologique } & \multicolumn{2}{|c|}{ Rétention } \\
\hline & & & $\mathrm{Ca}$ & $\mathbf{P}$ \\
\hline & $\%$ & $\%$ & $\%$ & \\
\hline Période $\mathrm{A}$. & 40,3 & $43, \mathrm{I}$ & 90,0 & 97,6 \\
\hline & $3 \mathrm{I}, \mathrm{I}$ & 27,2 & 94,0 & 82,6 \\
\hline$\ldots \ldots \ldots \ldots \ldots$ & 29,6 & 28,7 & 95,9 & 84,5 \\
\hline
\end{tabular}

La suppression du supplément minéral le $5^{\mathrm{e}}$ jour de la période $\mathrm{D}$ provoque un accroissement immédiat du rapport $\frac{\mathrm{Ca}}{\mathrm{P}}$ del'urine. En revanche on n'observe pas ce phénomène pendant la période $F$; peut-être faut-il 
en voir la raison dans l'existence d'une certaine réserve de calcium provoquée par les régimes alimentaires des périodes précédentes.

L'existence d'une forte excrétion urinaire de phosphore, lorsque le régime est lui-même riche en cet élément, a été signalée par différents auteurs. HALDI et coll. (9) voient, au cours d'expériences sur le rat, le phosphore de l'urine considérablement augmenté lorsque les taux de Ca et $\mathrm{P}$ du régime de base sont multipliés respectivement par 2,5 et 3,2, alors que l'excrétion urinaire de $\mathrm{Ca}$ demeure inchangée. AxELSSON (IO) indique que l'excrétion de calcium urinaire du mouton n'est pas influencée par le taux de calcium du régime, tandis qu'il observe une corrélation de $+0,4 \mathrm{I} 7$ entre les quantités de phosphore respectivement ingérées et éliminées par l'urine.

\section{b) Fixation quotidienne de phosphore et de calcium :}

Nous avons calculé les quantités totales de calcium et de phosphore fixées par les porcs pendant toute la durée de l'expérience, compte tenu des jours pendant lesquels les échantillons n'ont pas été récoltés et pour lesquels nous avons admis une fixation identique à celle observée pendant la période correspondante et compte tenu des jours auxquels correspondent des bilans négatifs. Le tableau VIII résume ces résultats.

\section{TABLEAU VIII}

\section{Fixation du $P$ et $C a$}

\begin{tabular}{|c|c|c|c|c|c|c|c|c|c|}
\hline & $\begin{array}{c}\mathrm{P} \\
\text { total }\end{array}$ & $\begin{array}{c}\mathrm{Ca} \\
\text { total }\end{array}$ & $\frac{\mathrm{Ca}}{\overline{\mathrm{P}}}$ & $\underset{\substack{\text { journa- } \\
\text { lier }}}{\mathrm{P}}$ & $\begin{array}{c}\text { Ca } \\
\text { journa- } \\
\text { lier }\end{array}$ & $\left|\begin{array}{c}\text { Gain } \\
\text { moyenne } \\
\text { de } \\
\text { poids vif }\end{array}\right|$ & $\begin{array}{l}\text { Poids } \\
\text { moyen }\end{array}$ & $\begin{array}{c}P(e+p) \\
\text { par kg } \\
\text { de } \\
\text { gain }\end{array}$ & $\begin{array}{l}\mathrm{Ca}(\mathrm{e}+\mathrm{p}) \\
\text { par kg } \\
\text { de } \\
\text { gain }\end{array}$ \\
\hline $\begin{array}{cc}\text { Porc } & 41 \ldots \ldots \\
\Rightarrow & 42 \ldots \ldots \\
; & 66 \ldots \ldots\end{array}$ & $\begin{array}{l}\mathrm{g} \\
\mathrm{I} 36,4 \\
\mathrm{IOI}, 2 \\
\mathrm{I} \mathbf{1 7}, 5\end{array}$ & $\begin{array}{c}g \\
310,5 \\
198,35 \\
23^{2,9}\end{array}$ & $\begin{array}{l}2,27 \\
\mathrm{I}, 96 \\
\mathrm{r}, 98\end{array}$ & $\begin{array}{c}\mathrm{g} \\
3,89 \\
2,89 \\
3,36\end{array}$ & $\begin{array}{l}\mathrm{g} \\
8,87 \\
5,66 \\
6,65\end{array}$ & $\begin{array}{l}g \\
512 \\
400 \\
426\end{array}$ & $\begin{array}{c}\mathrm{K} \\
50,6 \\
40,0 \\
43,9\end{array}$ & $\begin{array}{c}\mathrm{g} \\
7,6 \\
7,23 \\
7,89\end{array}$ & $\begin{array}{c}g \\
17,3 \\
14,15 \\
15,6\end{array}$ \\
\hline Moyennne.... & & & & 3,35 & 7,06 & 446 & 44,8 & 7,57 & I 5,7 \\
\hline
\end{tabular}

Observons en premier lieu que le rapport des taux de Ca et de $P$ fixés varie dans le même sens que les gains de poids quotidiens moyens. Ce rapport tend vers 2,3 , qui est la valeur correspondant à la matière osseuse (Bachmann et coll. (8)) (Polonovsky et Cartier 2,22 (II)). Le besoin d'entretien paraît donc négligeable par rapport au besoin de production chez l'animal dont la croissance est rapide. En conséquence, il semble qu'il y ait intérêt à fournir à l'animal une ration dont le rapport des éléments $\mathrm{Ca}$ et $\mathrm{P}$ fixés soit voisin de 2. 
Les fixations quotidiennes moyennes de $\mathrm{Ca}$ et de $\mathrm{P}$ ont été respectivement de 3,35 g et 7,06 g. Par kilogramme de gain de poids (besoins d'entretien inclus), les fixations journalières correspondantes se sont donc élevées à $7,57 \mathrm{~g}$ pour le $\mathrm{P}$ et à $\mathrm{r} 5,7 \mathrm{~g}$ pour le $\mathrm{Ca}$. Si l'on tient compte de l'utilisation physiologique trouvée précédemment (moyenne des périodes $\mathrm{B}$ et $\mathrm{C}$ ), on constate qu'il parait nécessaire de fournir environ $27 \mathrm{~g}$ de phosphore et $5^{2} \mathrm{~g}$ de calcium alimentaire par $\mathrm{kg}$ de gain de poids vif, ce qui correspond à un rapport $\frac{\mathrm{Ca}}{\mathrm{P}}$ égal à $\mathrm{I}, 9$. Si l'on calcule d'après ces données les besoins pratiques moyens des porcs de notre expérience, on constate qu'ils s'élèvent par jour à $\mathrm{I} 2 \mathrm{~g}$ de $\mathrm{P}$ et à $23,2 \mathrm{~g}$ de $\mathrm{Ca}$, soit environ respectivement $7,4 \mathrm{~g}$ et $14,3 \mathrm{~g}$ par $\mathrm{kg}$ d'aliment sec.

Ces valeurs sont notablement plus élevées que celles que l'on peut trouver dans la littérature antérieure. Les données américaines (I2) indiquent comme besoin pour un porc de $45 \mathrm{~kg}$ (croissance non précisée) I3,7 $\mathrm{g}$ de calcium et 9, I $\mathrm{g}$ de phosphore.

Or, nos propres données présentent l'avantage d'une concordance satisfaisante avec les observations concernant l'accroissement de poids quotidien du squelette des porcs. D'après les résultats de MCMEECKAN (I3), cet accroissement est sensiblement égal au I/ro du gain quotidien de poids vif. Pour des porcs qui s'accroissent quotidiennement de $45^{\circ} \mathrm{g}$, le poids de matière osseuse correspondant serait de $45 \mathrm{~g}$ environ. Nous savons, d'autre part, que la masse de substance minérale de cette matière osseuse est de $\mathrm{I} 8 \mathrm{~g}$ (résultats personnels non publiés). Sachant, d'autre part, que les cendres d'os contiennent environ I $8 \%$ de $\mathrm{P}$ et $37,6 \%$ de Ca (I4), nous voyons ainsi que les quantités fixées de ces éléments devraient être respectivement de $3,2 \mathrm{~g}$ de $\mathrm{P}$ et $6,8 \mathrm{~g}$ de $\mathrm{Ca}$, alors que nous obtenons expérimentalement $3,35 \mathrm{~g}$ de $\mathrm{P}$ et $7,06 \mathrm{~g}$ de $\mathrm{Ca}$.

Nous ne pouvons, toutefois, affirmer que la fixation de ces éléments eût été analogue si l'on avait utilisé d'autres phosphates ou des taux alimentaires de $\mathrm{P}$ et $\mathrm{Ca}$ différents.

En conclusion, le phosphate défluoré que nous avons étudié peut-être utilisé dans 1'alimentation des porcs. A la dose de $2,25 \%$ de la ration, il est convenablement assimilé et retenu par l'organisme de ces animaux.

\section{RESUMÉ ET CONCLUSIONS}

$\mathrm{I}^{0}$ L'utilisation digestive, le bilan physiologique et la rétention du phosphore et du calcium d'un phosphate naturel défluoré sont mesurés chez trois pores en croissance. Le phosphate est introduit dans les rations aux doses de $2,25 \%$ et $4,5 \%$. Nous enregistrons les variations quotidiennes des excrétions fécales et urinaires de phosphore et de calcium. 
$2^{\circ}$ Pour un même régime alimentaire, la teneur des matières fécales en $\mathrm{P}$ et en $\mathrm{Ca}$ est relativement constante. $\mathrm{L}_{\mathbf{L}}$ a composition de l'urine subit de plus grandes variations.

$3^{\circ}$ Les bilans physiologiques correspondant à la période témoin (sans phosphate) sont égaux à 40,3 pour le calcium et 43 , I pour le phosphore. Au cours des périodes correspondant aux régimes contenant $2,25 \%$ de phosphate défluoré, les valeurs moyennes correspondantes sont respectivement égales à 30,4 et 28 .

$4^{\circ}$ Les quantités moyennes de $\mathrm{Ca}$ et $\mathrm{P}$ fixées quotidiennement sont respectivement de $3,35 \mathrm{~g}$ et $7,06 \mathrm{~g}$ pour un gain moyen quotidien de poids de $446 \mathrm{~g}$ et un poids moyen de $44,8 \mathrm{~kg}$.

$5^{\circ}$ Les besoins pratiques en $\mathrm{P}$ et $\mathrm{Ca}$ par $\mathrm{kg}$ de matière sèche d'aliment peuvent être évalués d'après ces expériences à I4,3 $\mathrm{g}$ de calcium et $7,4 \mathrm{~g}$ de phosphore. Le rapport $\frac{\mathrm{Ca}}{\mathrm{P}}$ est donc, dans ce cas de $r, 9$.

$6^{\circ}$ Les résultats sont obtenus avec un phosphate naturel défluoré ; ils ne peuvent être généralisés sans étude complémentaire. Ils montrent que ce phosphate peut être incorporé pratiquement dans les rations alimentaires des porcs afin de couvrir les besoins en $\mathrm{P}$ et en $\mathrm{Ca}$ de ces animaux.

Reçu pour publication le 1er juillet 1953

\section{BIBLIOGRAPHIE}

(I) Jacquot-ARmand (Y.), Jacquot (R.) et BERTrand (J.). - Recherches sur 1'assimilation du calcium et du phosphore. - I. Influence de la nature des sels ingérés sur la rétention du calcium et du phosphore. Bull. Soc. Chim. Biol., 26, 57, I944.

(2) Randoin (I.), Hugot (D.), Causeret (J.) et Morel (G.). - Utilisation physiologique du calcium des calcaires dolomitiques et du calcium du carbonate de calcium. Bull. Soc. Chim. Biol., 32, 807, I950.

(3) McClure (F. J.) et Mitchel, (H. H.). - The effect of calcium fluoride and phosphate rock on the calcium retention of young growing pigs. J. of $A g r$. Res., 42, 363, I931.

(4) PERo (R.). - Expériences portant sur l'assimilation des phosphates tricalciques naturels finement moulus par l'organisme du porc. Ann. Agron., 15, 288, I945.

(5) Cahn (T.), Houget (J.) et Jacouot (R.). - Modifications chimiques accompagnant la contraction musculaire et l'hyperthermie. I. - Méthodes analytiques. Ann. Physiol., 9, 205, I933.

(6) ThivolLE (L.). - Le dosage de l'ion phosphorique par molybdomanganimétrie. Application de la détermination du phosphore total sanguin et des différentes fractions du phosphore acido-soluble. Bulletin des Biologistes pharmaciens, 42, 372, I938.

(7) LEROY (A. M.), LERY (G.). ZeLteR (S. Z.) - Contribution à l'étude de 1'utilisation digestive des pulpes de betteraves desséchées par les porcs et les ruminants. Ann. Zootechnie, 1, 29, I952.

(8) Bachmann (G.), Haldi (J.) Wynn (W.), Ensor (Ch.). - The effects produced by decreasing the calcium and phosphorus intake on $\mathrm{Ca}$ and $\mathrm{P}$ absorption 
(IV, I953) UTILISATION DIGESTIVE DU PHOSPHORE ET DU CAICIUM 301 and deposition on various bodily constituents of the rat. $J$. of Nutr., 20, I 45, I 940 .

(9) Haldi (J.), Bachmann (G.), Wynn (W.) et Ensor (Ch.). - The effects produced by an increase in the $\mathrm{Ca}$ and $\mathrm{Pa}$ content of the diet on the $\mathrm{Ca}$ and $\mathrm{P}$ balances and on various bodily constituents of the rat. $J$. of Nutr., 18, 399, I939.

(Io) AXELSSON (M.), FRIKSSON (S.). - Factors influencing the calcium and phosphorus metabolism of adult sheep. Ann. of Roy. Agr. Coll of Sweden, 16, 7II, I949.

(II) Polonovsky (M.) et Cartier (P.). - Le phosphore dans l'organisme. Bull. Soc. Sci. Hyg. Alim., 39, 238, I95I.

(I2) Aliman (R. T.) et HAMriton (T. S.). - Carences alimentaires du bétail. Etude Agricole de la F. A. O., n ${ }^{\circ}$ 5, Washington-Rome, r95o.

(I3) MCMEEKAN (C. P.). - Growth and development in the pig with special reference to carcass quality characters. $J$. of A gric. Sc., 30, 276, 1940.

(r4) Evans (R. E.). - The influence of a low and high calcium diet on the development and chemical composition of the skeleton in Swine. $J$. of Agr. Sc., 20, II7-I25, I930. 